\title{
Market sharing dynamics between two service providers
}

\author{
Olivier Gallay *, Max-Olivier Hongler
}

Ecole Polytechnique Fédérale de Lausanne (EPFL), STI-IPR-LPM, Station 17, CH-1015 Lausanne, Switzerland

Received 8 September 2006; accepted 17 June 2007

Available online 22 June 2007

\begin{abstract}
We study the market partition between two distinct firms that deliver services to waiting time sensitive customers. In our model, the incoming customers select a firm on the basis of its posted price, the expected waiting time and its brand. More specifically, we quantify by a cost any departure from the ideal brand expected by each incoming customer. Considering that the two underlying queueing processes operate under high traffic regimes, we analyze the market sharing dynamics by using a diffusion process. As a function of control parameters, such as the waiting and brand departure costs or the incoming traffic intensity, we are able to analytically characterize a transition between an Hotelling-like regime (dominated by brand considerations) and a deadline type regime (dominated by waiting time considerations). The market sharing dynamics is described by the time evolution of a boundary point, which time evolution belongs to the class of noise-induced phase transitions, so far widely discussed in physics, chemistry and biology. Explicit illustrations for both symmetric (i.e. identical servers) and asymmetric cases are worked out.
\end{abstract}

(C) 2007 Elsevier B.V. All rights reserved.

Keywords: Stochastic processes; Hotelling model; Heavy traffic queueing dynamics; Multiplicative noise; Noise-induced phase transition

\section{Introduction}

In his original contribution [7], Hotelling did consider the case where two vendors supply an identical product that is perceived homogeneous by incoming customers. However, the vendors being separated in geographical space, transportation costs to be added to the mill prices charged by the vendors are generated. In presence of two vendors, it exists an inner market boundary point, for which the mill price plus the transportation costs from both suppliers break even. This seminal modeling framework has stimulated a wealth of contributions with the goal to relax some of the oversimplifying hypothesis of the original model. In particular, the introduction of elastic demands (i.e. customers are not prepared to pay "prohibitive prices" for the product) has been discussed in [10]. Note that the original Hotelling's model is basically deterministic - it indeed does not incorporate random perturbations which actually may corrupt the prices and then affect the customers' decision process. Among the numerous potential noise sources, one of the simplest and most

\footnotetext{
${ }^{*}$ Corresponding author. Tel.: +4121 6935817; fax: +41216933891.

E-mail address: olivier.gallay@epfl.ch (O. Gallay).
} 
natural way to incorporate randomness is to consider the situations where the customers' decision to select one of the vendors depends on the expected time delay before service. This simple and realistic situation has been recently proposed by Cachon and Harker in [2,3]. As these authors clearly emphasized in [2], the resulting inherent analytical complexity implies that rather seldom are the models dealing with firms that simultaneously compete with both prices and processing rates. The aim of this note is to investigate a class of simple models for which explicitly analytical considerations can partly be worked out. While in [2] the firms are assumed to adjust their processing rates to guarantee a fixed expected time cost, our class of models takes into account the fluctuations of the waiting times and therefore keeps full track of the randomness induced by the underlying queueing processes. Note that the adjusting processing rates assumption proposed in [2] allows a discussion based only on averages. Contrary to [2], where no variance effects enter into the description of the model (i.e. this is effectively a "pseudo-stochastic" model), our approach explicitly emphasizes the role played by the fluctuations variance - also called in the sequel the "volatility" of the underlying noise sources. As discussed in [5], the introduction of waiting costs in the queueing dynamics leads to the concept of externalities (i.e. the costs induced on later incomers by a customer who is just joining the queue). In the class of models to be discussed here, these externalities trigger the random dynamics controlling the boundary point which defines the market partition. While, for Hotelling-like models, the interest is paid directly on the competition between the servers (see for instance $[1,3,10,11]$ ), in the present study we exclusively focus on the market sharing dynamics.

Service models where distance and quality of service enter into consideration find, among others, a practical framework in the secondary health care market. More precisely, let us consider patients who wait for non-urgent operations, that can be mid-term planed. As said in [9], where an application of the standard Hotelling model to the secondary health care market is proposed, patients may accept meeting monetary and non-monetary costs inherent to distance, if they expect a positive return in term of enhanced quality of service. Furthermore, the quality of service perceived by the patients combines different aspects, including the time to wait for the operation to take place. Another typical situation will be met when car drivers entering into a city center are offered alternative choices between several parking lots (here we focus on two lots). It is nowadays common to post in real time, at the entrance of the city, the number of available parking spaces of each parking lot. The actual time required to complete a parking action, which here plays the role of the waiting time, is clearly monotonously decreasing with the number of available spaces of the parking lot. Hence, the selection of the best parking lot does not only depend on its location, but also on its current content.

In Section 2, we introduce the two service providers linear market considered throughout this paper. In Section 3, attention is restricted to the simplest case where symmetric configurations are discussed. We show that, for heavy traffic regimes of the underlying queueing processes, the boundary point partitioning the market interval is governed by a scalar stochastic differential equation with multiplicative noise source. For this dynamics, it is possible to explicitly calculate the associated stationary probability measure. The multiplicative character of the noise source offers the possibility to observe a so-called noise-induced phase transition, which manifests itself by a change of the modal character of the stationary probability measure - a transition from a uni- to a bimodal probability density. In the present context, the transition between these two regimes relates to a transition between a regime where the Hotelling's spatial (i.e. the brand) character dominates in the decision taken by the incoming customers and a regime where the time delays dominate. We explicitly work out a simple, though fully representative, illustration belonging to our class of models. For this particular case, we are able to fully calculate the relaxation rate (i.e. the transient regime) characterizing the approach towards the final statistical equilibrium. The relaxation process is strongly dependent on the relative importance of the externalities arising in the associated queueing processes. A short account devoted to simulation experiments explicitly comforts our analytical findings. The dynamics arising for asymmetric configurations is discussed in Section 4. Following the technique used for the symmetric case, we compute the stationary probability density function of the boundary point when the two servers work at different service rates (i.e. dynamic asymmetry). We also consider the cases where the two service providers charge non-equal prices and the configurations where the two servers do not have symmetric positions with respect to the center of the market. We show that while these static asymmetries strongly influence the transient regime, they however do not affect the stationary measure. 


\section{Model for market sharing dynamics}

As in [2], our starting point will be a two servers Hotelling's model where two service providers $S_{1}$ and $S_{2}$ are located in a (linear) market confined on a segment $\Omega:=[-\Delta,+\Delta] \subset \mathbb{R}, \Delta>0$. The positions of the service providers are denoted respectively by $x_{1}$ and $x_{2}$ and satisfy $x_{1}<x_{2}$. Let $L=x_{2}-x_{1}$ denotes the distance between $S_{1}$ and $S_{2}$. The servers $S_{1}$ and $S_{2}$ charge respectively prices $p_{1}$ and $p_{2}$. Departing now from the original Hotelling's model, we add queueing processes in front of $S_{1}$ and $S_{2}$ and following [5], we will attach waiting costs to any customer lining in the queues before being served. Taking into account waiting costs thus confers a dynamical character to the original Hotelling's model. Specifically, our dynamic model exhibits the following features and obeys to the following rules:

(a) Arrivals dynamics. Incoming customers follow a Poisson rule with rate $\Lambda$, hence the average time between two arrivals will be $\Lambda^{-1}$.

(b) Spatial distribution of the arrivals. Incoming customers arrive at a random location $x \in \Omega$ drawn from a uniform probability density $U(\Omega)$ with support on $\Omega$.

(c) Services dynamics. Both servers $S_{i}, i=1,2$, have generally distributed service times with rate $\mu_{i}$, hence the average service time will be $\mu_{i}^{-1}, i=1,2$.

(d) Traffic intensity. The traffic into the system is limited to $\rho:=\frac{1}{\mu_{1}+\mu_{2}}<1$. This ensures that the system is globally stable, i.e. the global incoming rate is less than the global service rate.

(e) Queueing processes. When an incoming customer finds both $S_{1}$ and $S_{2}$ busy, he/she will wait for service and line-up in a queue. The capacity of the queue is assumed to be unlimited and the service discipline is first-in-first-out (FIFO). In view of points (a) and (c), we hence consider M/G/1 queues.

(f) Customer information gathering. Upon his/her arrival at $x \in \Omega$, each incoming customer knows:

(1) his/her relative distance $\left|x-x_{1}\right|$ and $\left|x-x_{2}\right|$ to $S_{1}$ and $S_{2}$.

(2) the contents $N_{1}(t)$ and $N_{2}(t)$ of both queues $\left(t \in \mathbb{R}^{+}\right.$being the arrival time). In other words, both queue contents are observable to any incoming customer.

(g) Cost structures. There are two types of costs incurred by any customer, namely:

(1) the waiting time cost (WTC), characterized by a cost parameter $c_{w}$ with physical unit [ $\left.\frac{\text { dollar }}{\text { time unit }}\right]$.

(2) the brand departure cost (BDC), quantified by a cost parameter $c_{t}$ with physical unit $\left[\frac{\text { dollar }}{\text { brand distance unit }}\right]$.

(h) Decision policy. Upon arrival, an incoming customer is aware of:

- the queue contents $N_{1}(t)$ and $N_{2}(t)$,

- his/her relative position to $S_{1}$ and $S_{2}$,

- the values of the costs $c_{w}$ and $c_{t}$,

- the service rates $\mu_{1}$ and $\mu_{2}$,

- the posted prices $p_{1}$ and $p_{2}$.

Based on this information set, the incoming customer decides which server $S_{1}$ or $S_{2}$ he/she will join.

(i) Demand structure. Following the original Hotelling's case, we assume an inelastic demand, i.e. a customer will purchase the service at any price, even if the proposed price is arbitrarily large.

A sketch of our modeling framework can be found in Fig. 1. Extending original Hotelling's configuration, waiting times confer to the above class of models an explicit dynamic character.

When served by $S_{i}$, an incoming customer feels a utility function $U_{i}(x), i=1,2$, where $x$ is the customer's initial position which enters into the decision policy. In words, the functions $U_{i}(x)$ quantify the gain realized by a customer choosing server $S_{i}$ when he/she enters at location $x$. Specifically, for linear waiting and transportation costs, the utility functions read as

$$
U_{i}(x)=a-p_{i}-c_{t}\left|x-x_{i}\right|-c_{w} \mathbb{E}\left(W_{i} \mid N_{i}(t)\right), \quad i=1,2,
$$

with $a$ being a systematic reward due to the service and $\mathbb{E}\left(W_{i} \mid N_{i}(t)\right)$ standing for the conditional expected waiting time at $S_{i}$ when $N_{i}(t)$ already waiting customers are observed. As $\mu_{i}^{-1}$ is the average service time at server $S_{i}$, this last conditional expectation is readily given by 


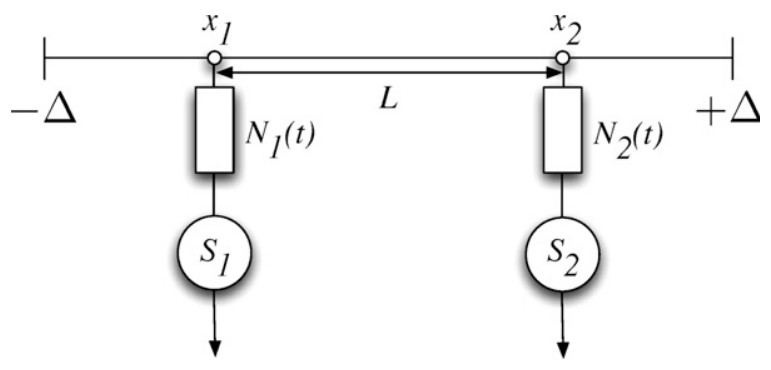

Fig. 1. Bounded market with two vendors and time sensitive customers.

$$
\mathbb{E}\left(W_{i} \mid N_{i}(t)\right)=\frac{N_{i}(t)}{\mu_{i}} .
$$

We obviously assume that any customer does maximize his utility function when choosing one of the two servers. This suggests to introduce a time-dependent boundary position $Y_{t} \in[-\Delta,+\Delta]$ implicitly defined by

$$
U_{1}\left(Y_{t}\right)=U_{2}\left(Y_{t}\right) \text {. }
$$

Hence, our strictly increasing BDC which we assume from now on imply that $Y_{t}$ dynamically separates the two monopolies held by $S_{1}$ and $S_{2}$. A sketch of the situation is given in Fig. 2. As $Y_{t}$ is a function of the two stochastic processes $N_{1}(t)$ and $N_{2}(t)$, it will be itself a stochastic process.

Let $\lambda_{i}\left(t, Y_{t}\right)$ denotes the partial incoming rate of customers feeding $S_{i}$ at time $t$ and hence:

$$
\lambda_{1}\left(t, Y_{t}\right)+\lambda_{2}\left(t, Y_{t}\right)=\Lambda, \quad \forall t \in \mathbb{R}^{+} .
$$

In view of the assumption (b) (i.e. spatially uniform arrival on $\Omega=[-\Delta,+\Delta]$ ), the partial traffic flows feeding $S_{1}$ and $S_{2}$ result from the Bernoulli "thinning" of the incoming Poisson flow with global rate $\Lambda$. The branching probability is given by $P=\frac{\Delta-Y_{t}}{2 \Delta}$ and it is established [4] that the thinning produces two independent Poisson processes with partial rates:

$$
\lambda_{1}\left(t, Y_{t}\right)=\frac{\Delta+Y_{t}}{2 \Delta} \Lambda \quad \text { and } \quad \lambda_{2}\left(t, Y_{t}\right)=\frac{\Delta-Y_{t}}{2 \Delta} \Lambda
$$

For the utility functions given by Eq. (1), the time-dependent boundary point will obey, $\forall t \in \mathbb{R}^{+}$:
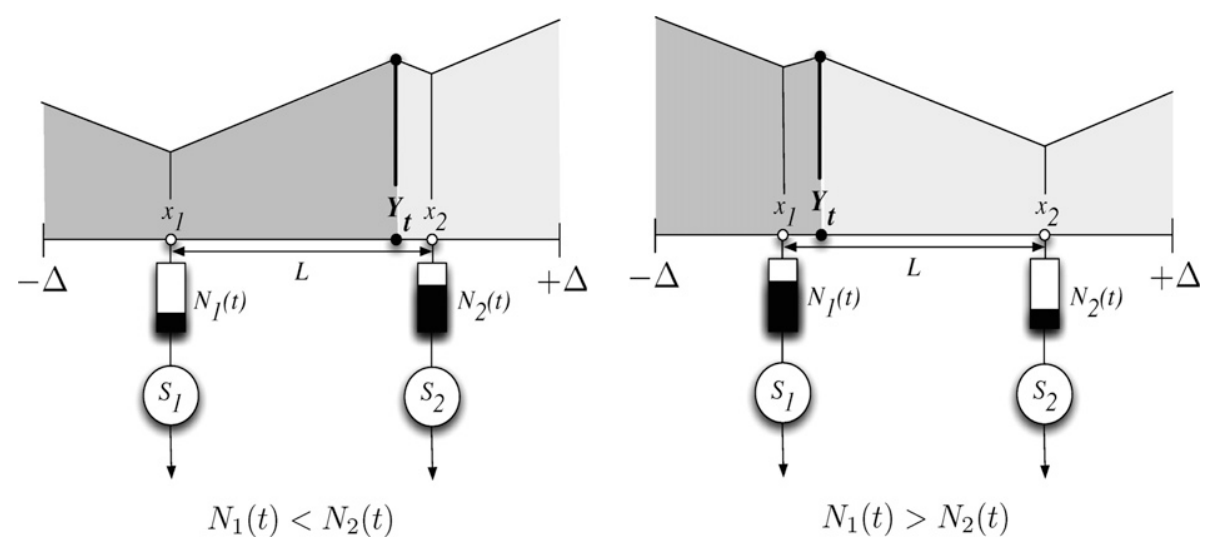

Fig. 2. Cost structure as a function of the customers' location. The total costs for a customer located at position $x$ are the sum of the selling price $p_{i}$, the waiting time $\operatorname{cost} c_{w} \mathbb{E}\left(W_{i} \mid N_{i}(t)\right)$ (identical service rates are assumed in this figure) and the brand departure cost $c_{t}\left|x-x_{i}\right|$. Any customer chooses the service provider minimizing his/her total costs (i.e. it corresponds to maximize his/her utility function). As a consequence, all the customers standing on the left of $Y_{t}$ will choose $S_{1}$, those on the right go to $S_{2}$. The difference between the two figures is the current queue contents. These contents determine the position of the boundary point $Y_{t}$, which separates the respective market shares held by $S_{1}$ and $S_{2}$. 


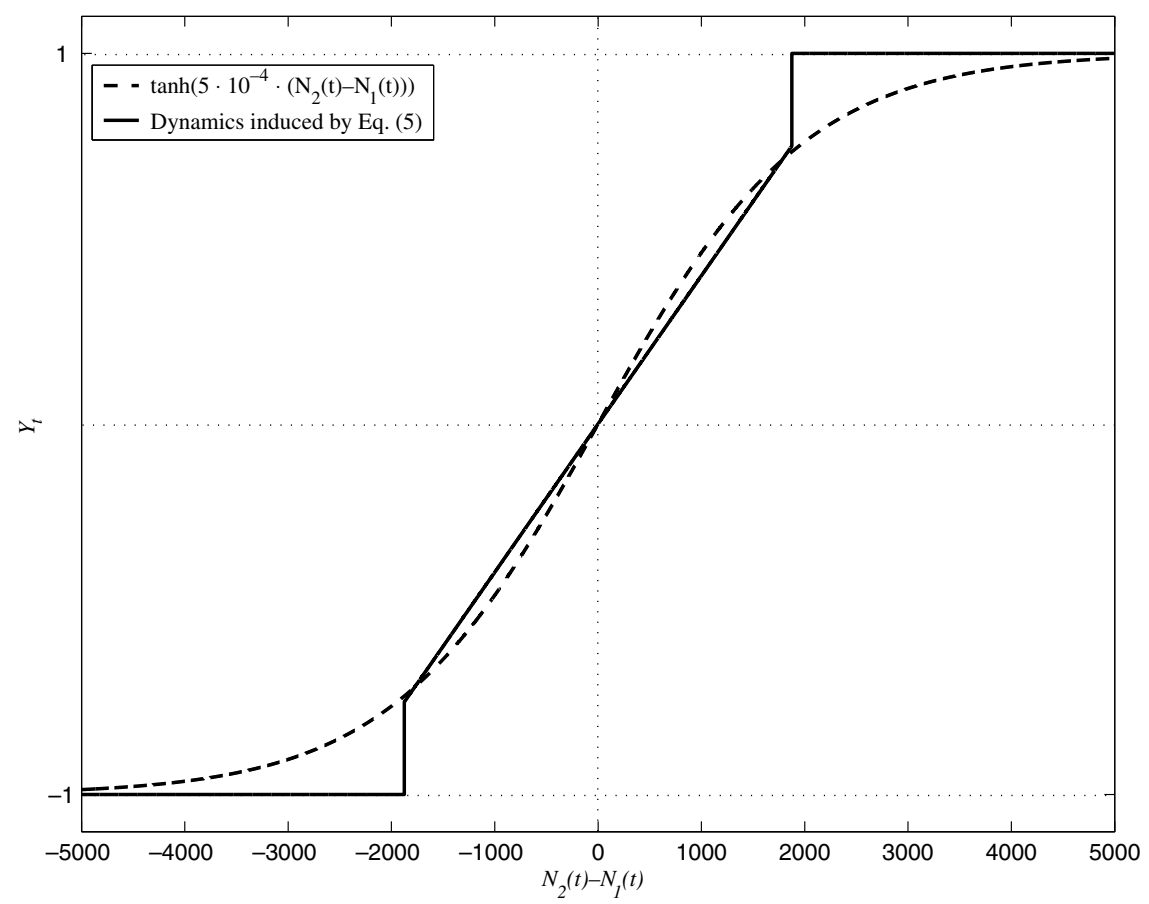

Fig. 3. Typical representation of the boundary position dynamics for $\Delta=1$. The solid line shows the dynamics induced by Eq. (5) when $c_{t}=10, c_{w}=8 \times 10^{-3}, \mu_{1}=\mu_{2}=1 p_{1}=p_{2}=1$ and $x_{2}=-x_{1}=\frac{3}{4}$ (symmetric configuration). The dashed line shows the dynamics given by Eq. (21) when $\gamma=5.33 \times 10^{-4}$.

$$
Y_{t}= \begin{cases}\text { (a) } \frac{c_{w}}{2 c_{t}}\left(\frac{N_{2}(t)}{\mu_{2}}-\frac{N_{1}(t)}{\mu_{1}}\right)+\frac{x_{1}+x_{2}}{2}+\frac{p_{2}-p_{1}}{2 c_{t}} & \text { if } c_{t} L \geqslant|\Psi| \\ (\mathrm{b})+\Delta & \text { if } c_{t} L<\Psi \\ (\mathrm{c})-\Delta & \text { if } c_{t} L<-\Psi\end{cases}
$$

where

$$
\Psi:=\Psi\left(N_{1}(t), N_{2}(t), \mu_{1}, \mu_{2}, p_{1}, p_{2}\right)=p_{2}-p_{1}+c_{w}\left(\frac{N_{2}(t)}{\mu_{2}}-\frac{N_{1}(t)}{\mu_{1}}\right) .
$$

In case (a), $Y_{t} \in\left[x_{1}, x_{2}\right] \subset[-\Delta,+\Delta]$. Indeed in this case, the BDC from one server to the other (i.e. $c_{t} L$ ) is greater than the global difference between the prices and the WTC's of the two servers (i.e. $|\Psi|)$. Hence, a customer located near the server having the longest queue will choose this server anyway. In cases (b) and (c), any customer in the whole interval $[-\Delta,+\Delta]$ joins the server having the shortest queue. Indeed, the gain in WTC (due to the difference between the queue contents) and in price exceeds the BDC incurred by the distance from one server to the other. A representation of the dynamics induced by Eq. (5) for a particular choice of the control parameters is found in Fig. 3.

We now separately discuss the symmetric and the asymmetric configurations.

\section{Symmetric configurations}

The positions of the service providers are assumed to satisfy $-\Delta \leqslant x_{1} \leqslant 0$ and $0 \leqslant x_{2} \leqslant+\Delta$ and they are located symmetrically with respect to the center of the market, i.e. $x_{1}=-x_{2}$. Furthermore, the servers $S_{1}$ and $S_{2}$ offer homogenous services $\mu_{1}=\mu_{2}=\mu$ and both charge an equal price $p_{1}=p_{2}=p$.

Let $A_{i}(t), D_{i}(t)$ and $N_{i}(t)$ respectively denote the numbers of arrivals, departures and the population in $S_{i}$ at time $t$. From now on, we restrict ourselves to heavy traffic regimes characterized by $\rho=\frac{1}{2 \mu}=1-\epsilon$, with $\epsilon$ small. Writing 


$$
N_{i}(t)=A_{i}(t)-D_{i}(t)
$$

in heavy traffic the server $S_{i}$ has very long busy period and hence the process $N_{i}(t)$ does almost never vanish, $i=1,2$. This implies that the departure and arrival processes are almost independent. In heavy traffic regimes, it is well established (see in particular [8]) that both queue contents at time $t$ are well approximated by diffusion processes of the form:

$$
N_{i}(t)=\int_{0}^{t}\left[\lambda_{i}\left(s, Y_{s}\right)-\mu\right] \mathrm{d} s+\int_{0}^{t} V_{i}\left(s, Y_{s}\right) \mathrm{d} B_{i, s}, \quad i=1,2,
$$

where $B_{1, t}$ and $B_{2, t}$ are independent standard Brownian motions and the terms $V_{i}\left(t, Y_{t}\right)$ denote the state-dependent "volatilities" given by

$$
V_{i}\left(t, Y_{t}\right)^{2}=\lambda_{i}\left(t, Y_{t}\right)^{3} \sigma_{a, i}^{2}+\mu^{3} \sigma_{s, i}^{2}, \quad i=1,2,
$$

with $\sigma_{a, i}^{2}$ (respectively $\sigma_{s, i}^{2}$ ) being the variance of the inter-arrival times (respectively the variance of the service times) for server $S_{i}$. Using Eqs. (3)-(7) and the fact that $B_{1, t}$ and $B_{2, t}$ are independent, we therefore can write:

$$
N_{2}(t)-N_{1}(t)=-\frac{\Lambda}{\Delta} \int_{0}^{t} Y_{s} \mathrm{~d} s+\int_{0}^{t} V\left(s, Y_{s}\right) \mathrm{d} B_{s}
$$

with $B_{t}$ being a standard Brownian motion and $V^{2}\left(t, Y_{t}\right)=V_{1}\left(t, Y_{t}\right)^{2}+V_{2}\left(t, Y_{t}\right)^{2}=\Lambda+\mu^{3}\left(\sigma_{s, 1}^{2}+\sigma_{s, 2}^{2}\right)-$ remember that for Poisson processes, we have $\sigma_{a, i}^{2}=\lambda_{i}\left(t, Y_{t}\right)^{-2}$.

In this symmetric configuration, Eq. (5) reduces to, $\forall t \in \mathbb{R}^{+}$:

$$
Y_{t}= \begin{cases}\frac{c_{w}}{2 \mu c_{t}}\left(N_{2}(t)-N_{1}(t)\right) & \text { if } c_{t} L \geqslant|\widetilde{\Psi}|, \\ +\Delta & \text { if } c_{t} L<\widetilde{\Psi} \\ -\Delta & \text { if } c_{t} L<-\widetilde{\Psi}\end{cases}
$$

where, for this symmetric case, we define:

$$
\widetilde{\Psi}=\Psi\left(N_{1}(t), N_{2}(t), \mu, \mu, p, p\right)=\frac{c_{w}}{\mu}\left(N_{2}(t)-N_{1}(t)\right) .
$$

To proceed further with analytical calculations, we approximate the dynamics implied by Eq. (9) by introducing an odd (due to the symmetry of the problem), effective monotonously increasing one-to-one, $C^{2}(\mathbb{R})$ function:

$$
f(\cdot): \mathbb{R} \rightarrow[-1,+1]
$$

fulfilling

$$
Y_{t}=\Delta f\left(\gamma\left(N_{2}(t)-N_{1}(t)\right)\right),
$$

with

$$
\gamma:=\frac{c_{w}}{\mu L c_{t}} .
$$

The control parameter $\gamma$ is dimensionless and quantifies the respective importance of the different costs. Note that in Eq. (12), the time unit is measured in average service time.

As $f$ is invertible, Eq. (11) can be written as

$$
f^{-1}\left(\frac{Y_{t}}{\Delta}\right)=\gamma\left(N_{2}(t)-N_{1}(t)\right) .
$$

Using Eq. (8), then Eq. (13) becomes

$$
f^{-1}\left(\frac{Y_{t}}{\Delta}\right)=-\frac{\gamma \Lambda}{\Delta} \int_{0}^{t} Y_{s} \mathrm{~d} s+\gamma \int_{0}^{t} V\left(s, Y_{s}\right) \mathrm{d} B_{s} .
$$


Differentiating, we obtain:

$$
\left(f^{-1}\right)^{\prime}\left(\frac{Y_{t}}{\Delta}\right) \mathrm{d} Y_{t}=-\gamma \Lambda Y_{t} \mathrm{~d} t+\Delta \gamma V\left(t, Y_{t}\right) \mathrm{d} B_{t}
$$

which can be written as

$$
\mathrm{d} Y_{t}=-\frac{\gamma \Lambda Y_{t}}{\left(f^{-1}\right)^{\prime}\left(\frac{Y_{t}}{\Delta}\right)} \mathrm{d} t+\frac{\Delta \gamma V\left(t, Y_{t}\right)}{\left(f^{-1}\right)^{\prime}\left(\frac{Y_{t}}{\Delta}\right)} \mathrm{d} B_{t} .
$$

In our settings (remember that we deal with $M / G / 1$ queues), $V\left(t, Y_{t}\right)=V=\sqrt{\Lambda+\mu^{3}\left(\sigma_{s, 1}^{2}+\sigma_{s, 2}^{2}\right)}$ does not depend on $Y_{t}$ nor on $t$. We can thus write Eq. (16) as

$$
\mathrm{d} Y_{t}=-\frac{\gamma \Lambda Y_{t}}{\left(f^{-1}\right)^{\prime}\left(\frac{Y_{t}}{\Delta}\right)} \mathrm{d} t+\frac{\Delta \gamma V}{\left(f^{-1}\right)^{\prime}\left(\frac{Y_{t}}{\Delta}\right)} \mathrm{d} B_{t}
$$

The stochastic differential equation (SDE) given by Eq. (17) describes the effective dynamics of the boundary position $Y_{t}$. The White Gaussian noise $\mathrm{d} B_{t}$ being merely the limit of finitely correlated processes, we assign to the underlying stochastic integral relative to Eq. (17) the Sratonovitch's interpretation [6]. Hence, the transition probability density $P\left(y, t \mid y_{0}, t_{0}\right)$ describing the solution of the $\operatorname{SDE}(17)$ reads as

$$
\frac{\partial}{\partial t} P\left(y, t \mid y_{0}, t_{0}\right)=\mathscr{F} P\left(y, t \mid y_{0}, t_{0}\right),
$$

with Fokker-Planck operator taking here the form, [6]:

$$
\mathscr{F}(\cdot):=\frac{\partial}{\partial y}\left[\frac{\gamma \Lambda y}{\left(f^{-1}\right)^{\prime}\left(\frac{y}{4}\right)}(\cdot)\right]+\frac{1}{2} \frac{\partial}{\partial y}\left[g(y) \frac{\partial}{\partial y} g(y)(\cdot)\right], \quad g(y)=\frac{\Delta \gamma V}{\left(f^{-1}\right)^{\prime}\left(\frac{y}{\Delta}\right)} .
$$

The stationary probability density function $P_{s}(y)$ solving Eq. (18), with vanishing left hand side, reads as

$$
P_{s}(y)=\mathscr{N}\left(f^{-1}\right)^{\prime}\left(\frac{y}{\Delta}\right) \exp \left\{-\frac{2 \Lambda}{\gamma \Delta^{2} V^{2}} \int^{y} u\left(f^{-1}\right)^{\prime}\left(\frac{u}{\Delta}\right) \mathrm{d} u\right\}
$$

for $y \in[-\Delta,+\Delta]$, with $\mathscr{N}<\infty$ a normalization constant.

Symmetry (i.e. our assumptions that $x_{1}=-x_{2}, \mu_{1}=\mu_{2}$ and $\left.p_{1}=p_{2}\right)$ implies that $P_{s}(y)=P_{s}(-y)$. In particular, studying the curvature $\mathscr{R}(0)$ of $P_{s}(y)$ at $y=0$ directly furnishes information regarding the modularity of $P_{s}(y)$. From Eq. (19), we directly obtain:

$$
\operatorname{sign}\{\mathscr{R}(0)\}=\operatorname{sign}\left\{-\gamma V^{2} f^{\prime \prime \prime}(0)-2 \Lambda\left(f^{-1}\right)^{\prime}(0)\left(f^{\prime}(0)\right)^{3}\right\} .
$$

For given functions $f$, we observe that the sign of the curvature $\mathscr{R}(0)$ directly depends on the values of the (control) external parameters (here $c_{w}, c_{t}, L, \Lambda$ and $\mu$ ) solely. A curvature sign change exhibits a transition of regime triggered by the presence of fluctuations. This is referred as a noise-induced phase transition [6] and an explicit illustration is now worked out.

\subsection{Explicit illustration - symmetric case}

Belonging to the previous class of models, the particular choice

$$
Y_{t}=\Delta \tanh \left(\gamma\left(N_{2}(t)-N_{1}(t)\right)\right)
$$

leads to very simple algebra. A particular representation of Eq. (21), put into comparison with the dynamics induced by Eq. (5), is found in Fig. 3.

For this particular case, the SDE (17), describing the effective boundary point dynamics, becomes:

$$
\mathrm{d} Y_{t}=-\gamma \Lambda Y_{t}\left(1-\left(\frac{Y_{t}}{\Delta}\right)^{2}\right) \mathrm{d} t+\Delta \gamma V\left(1-\left(\frac{Y_{t}}{\Delta}\right)^{2}\right) \mathrm{d} B_{t}
$$

In view of Eq. (19), the corresponding stationary probability density function simply becomes: 


$$
P_{s}(y)=\mathscr{N}\left(1-\left(\frac{y}{\Delta}\right)^{2}\right)^{\frac{1}{\gamma V^{2}}-1} \text { for } y \in[-\Delta,+\Delta],
$$

where $\mathscr{N}$ is the normalization constant given here by

$$
\mathscr{N}^{-1}=\Delta \int_{0}^{1} t^{-\frac{1}{2}}(1-t)^{\frac{1}{\gamma^{2}}-1} \mathrm{~d} t=\Delta B\left(\frac{1}{2}, \frac{\Lambda}{\gamma V^{2}}\right)
$$

where $B(x, y):=\frac{\Gamma(x) \Gamma(y)}{\Gamma(x+y)}$ and $\Gamma(x)$ stands for the Gamma function. An illustration of the probability density function given by Eq. (23) for different values of $\gamma$ and $\Delta=1$ is found in Fig. 4. Regarding Eq. (20), the sign of the curvature $\mathscr{R}(0)$ of $P_{s}(y)$ at $y=0$ is here given by

$$
\mathscr{R}(0) \begin{cases}>0 & \text { when } \frac{1}{\gamma V^{2}}<1, \\ =0 & \text { when } \frac{1}{\gamma V^{2}}=1, \\ <0 & \text { when } \frac{1}{\gamma V^{2}}>1 .\end{cases}
$$

The information given by Eq. (24) (which is in perfect agreement with what we would expect with regard to the form of $P_{s}(y)$ given by Eq. (23)) perfectly describes the modularity of $P_{s}(y)$ and the underlying noise-induced phase transition.

\subsubsection{Transient behavior}

For the choice given in Eq. (21), we can also study the rate of approach to the equilibrium. Indeed, by introducing the change of variables:

$$
t \mapsto \tau=\gamma^{2} V^{2} t, \quad X_{t} \mapsto Y_{t}=\Delta \tanh \left(X_{t}\right),
$$

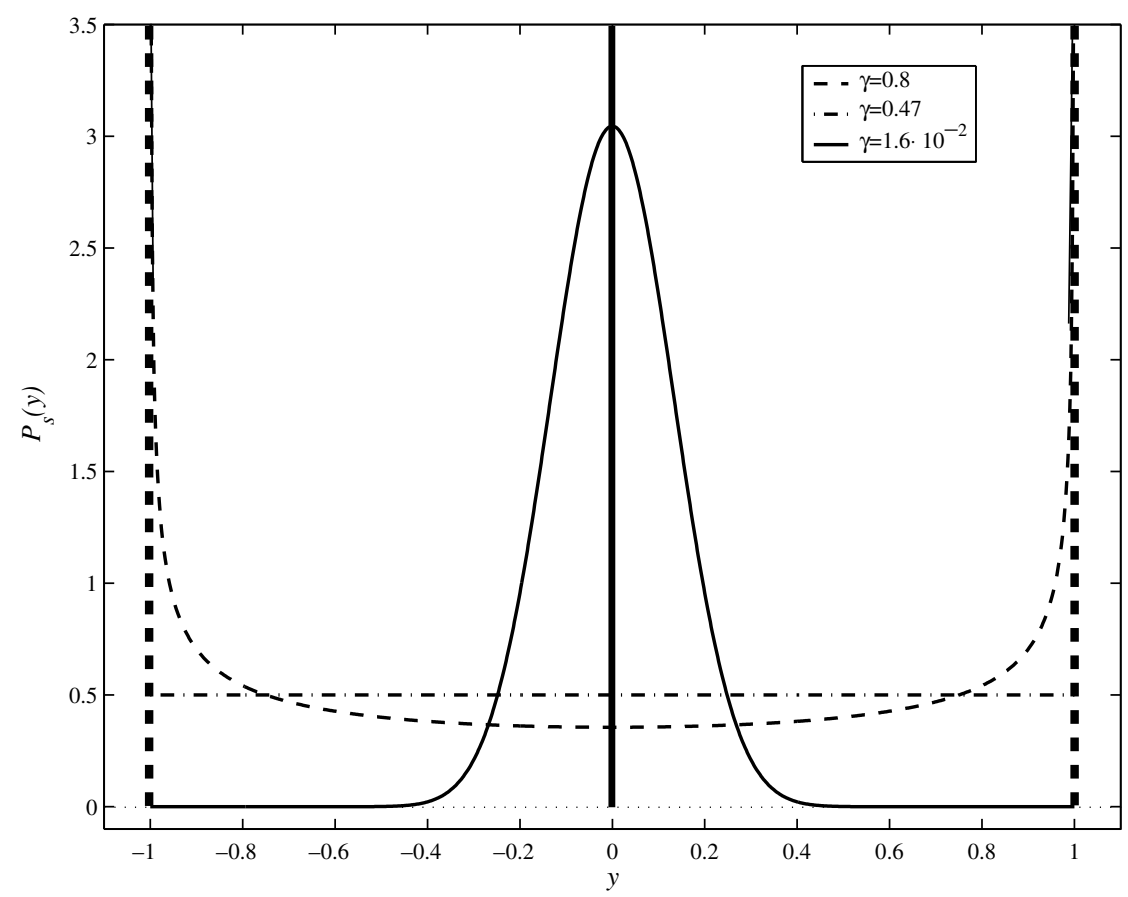

Fig. 4. Stationary probability density function of the time-dependent boundary position $Y_{t}$ when $\Delta=1, \Lambda=1.8, \mu=1(\rho=0.9)$ and the service time processes are Poisson. This density is drawn for three different values of $\gamma=\left[0.8 ; 0.47 ; 1.6 \cdot 10^{-2}\right]$. Furthermore, when $\gamma \rightarrow \infty$ (it corresponds to purely deadline type regimes), the density is sharply peaked at $y=-\Delta=-1$ and $y=+\Delta=+1$. In the other limit, $\gamma \rightarrow 0$ (corresponding to purely Hotelling-like regimes), the density is restricted to a single peak at $y=0$. This graph clearly exhibits the noise-induced phase transition arising in our dynamic model. 
the dynamics given by Eq. (22) reduces to

$$
\mathrm{d} X_{\tau}=-\left(\frac{\Lambda}{\gamma V^{2}}+1\right) \tanh \left(X_{\tau}\right) \mathrm{d} \tau+\mathrm{d} W_{\tau}:=-2 K \tanh \left(X_{\tau}\right) \mathrm{d} \tau+\mathrm{d} W_{\tau}
$$

and the time-dependent solution $P\left(y, t \mid y_{0}, 0\right)$ of the associated Fokker-Planck is known for long (see for instance [12]). As an illustration, let us mention that for the situations where the dimensionless parameter $K:=\frac{\Lambda}{2 \gamma V^{2}}+\frac{1}{2} \in \mathbb{N}$, the explicit form simplifies somewhat and is given by [12]

$$
\begin{aligned}
P\left(x, t \mid x_{0}, 0\right)= & \frac{1}{1+z^{2}}\left[\left(1+z_{0}^{2}\right)\left(1+z^{2}\right)^{\frac{K}{2}} \frac{1}{2 \sqrt{\pi \tau}} \mathrm{e}^{-K^{2} \tau} \mathrm{e}^{-\frac{\left(x-x_{0}\right)^{2}}{4 \tau}}\right] \\
& +\frac{1}{\pi\left(1+z^{2}\right)} \sum_{n=0}^{K-1} \frac{(K-n)}{n ! \Gamma(2 K+1-n)} \mathrm{e}^{-n(2 K-n) \tau} \theta_{n}\left(x_{0}\right) \theta_{n}(x) f_{n}\left(x, x_{0}, t\right),
\end{aligned}
$$

with the definitions:

$$
\sinh (z):=y, \quad f_{n}\left(x, x_{0}, t\right):=\frac{1}{\sqrt{\pi}} \int_{g\left(x, x_{0}, t\right)-(K-n) \sqrt{t}}^{g\left(x, x_{0}, t\right)+(K-n) \sqrt{t}} e^{-u^{2}} \mathrm{~d} u, \quad g\left(x, x_{0}, t\right):=\frac{\operatorname{arcsinh}(x)-\operatorname{arcsinh}\left(x_{0}\right)}{2 \sqrt{t}}
$$

and the polynomials:

$$
\theta_{n}(x):=(-1)^{n} 2^{K-n} \Gamma\left(K-n+\frac{1}{2}\right)\left(1+x^{2}\right)^{K+\frac{1}{2}} \frac{\mathrm{d}^{n}}{\mathrm{~d} x^{n}}\left(1+x^{2}\right)^{n-K-\frac{1}{2}} .
$$

In particular, the long time scale $t_{\text {relax }}$ governing the approach to the stationary state given by Eq. (23) is determined by the spectral gap between 0 and the first non-vanishing eigenvalue of the Fokker-Planck Eq. (18) (remember that the vanishing eigenvalue corresponds to the stationary probability measure given by Eq. (19)). It follows that:

$$
1 / t_{\text {relax }}= \begin{cases}(2 K-1) \gamma^{2} V^{2}=\Lambda \gamma & \text { if } K \geqslant 1, \\ K^{2} \gamma^{2} V^{2}=\frac{\gamma \Lambda+\gamma^{2} V^{2}}{2} & \text { if } K<1\end{cases}
$$

From Eq. (28), we can draw the following remarks:

(a) Spectral characteristics of the Fokker-Planck equation. In view of Eq. (28), there are two relaxation regimes governed by the spectral properties of the associated Fokker-Planck Eq. (18). As discussed in [12], for $K \geqslant 1$ the spectrum exhibits both discrete and continuum parts whereas for $K<1$ only the continuum part survives.

(b) Regime transitions. Note that the transition from unimodal to bimodal densities given in Eq. (23) by $\left(\frac{1}{\gamma V^{2}}-1\right)=0$ coincides with the transition in the relaxation regimes given by Eq. (28).

(c) Rate of approach to the equilibrium. When discrete eigenvalues exist, the asymptotic time relaxation towards the single mode stationary probability density (given by Eq. (23)) is faster compared to the relaxation rate associated with the purely continuum spectrum which drives the system to the bimodal density (given by Eq. (23)). This can be intuitively understood in limiting regimes. Indeed, for the pure Hotelling's case, a situation arising when $c_{t} \rightarrow \infty$, the boundary position probability density is deltapeaked in the middle of the market interval (remember that we did focus in this section on symmetric configurations), and the relaxation time to reach this equilibrium is vanishingly small - this corresponds to the deterministic scheduling which commands to "join the closest server". For dominating Hotelling's type regimes, the externalities (i.e. the waiting costs affecting incomers arriving behind a customer entering into service) have little influence on the equilibrium probability density which describes the boundary point - this produces a fast relaxation towards the statistical equilibrium, which will be close to the limiting delta-peaked density. In the contrary, when the deadline type regime dominates, a new incomer strongly modifies the dynamical state of the system and hence strongly perturbs the underlying probability measure, thus implying long relaxation times to the statistical equilibrium. Note that for $K=0$ in Eq. (28), a situation realized when $c_{w} \rightarrow \infty$, the relaxation time diverges to infinity, meaning that no 


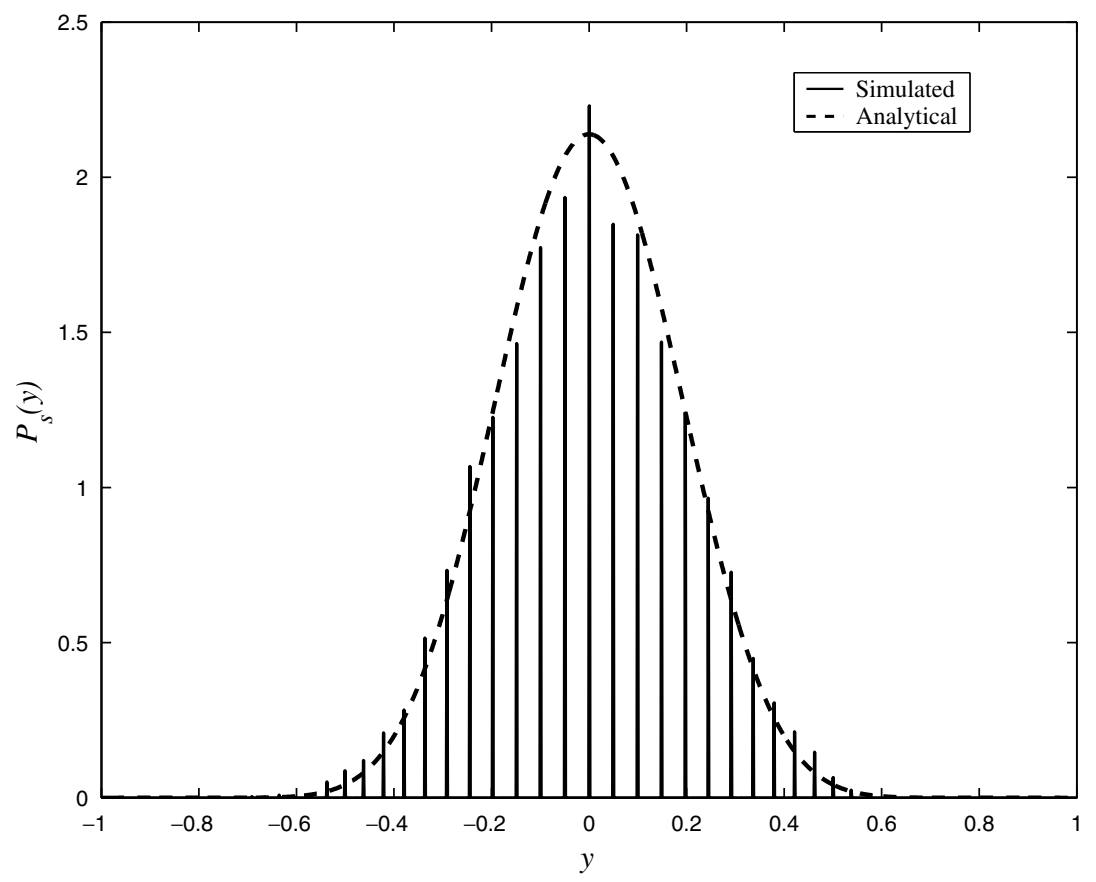

Fig. 5. Simulated and theoretical stationary probability density function of the time-dependent boundary position $Y_{t}=\Delta$. $\tanh \left(\gamma\left(N_{2}(t)-N_{1}(t)\right)\right)$ when $\Delta=1, \Lambda=1.9, \mu=1(\rho=0.95), \gamma=5 \cdot 10^{-2}$ and the service time processes are Poisson.

statistical equilibrium exists - this corresponds to the purely deterministic scheduling which commands to always "join the server exhibiting the shortest queue".

\subsubsection{Simulation experiments}

We have simulated the dynamics of the boundary position $Y_{t}$ in the particular case where $Y_{t}$ fulfills Eq. (21). Each customer, upon arrival, determines on which side of the boundary point $Y_{t}$ (dynamically given by Eq. (21), with regard to the current content of the queues) is his/her (uniformly distributed) position and he/she joins the queue hence chosen. We have computed an estimation of the stationary probability density function of the boundary position $Y_{t}$ after $10^{5}$ customers have passed through the system. The simulation experiments performed for different values of the control parameters (here $\gamma, \Lambda$ and $\mu$ ) confirm the presence of the noise-induced phase transition. The particular simulation results are compared with theoretical findings given by Eq. (23) (see Fig. 5).

\section{Asymmetric configurations}

Different sources of asymmetry are possible:

- Dynamic asymmetry. This situation is encountered when heterogeneous servers are operating, it is treated in Section 4.1.

- Static asymmetry. This arises in presence of non-symmetric server locations and non-equal prices. Configurations where the servers have asymmetric positions with respect to the center of the market and situations where the posted prices are different lead to analogous dynamics and are discussed in Section 4.2.

\subsection{Heterogeneous servers}

We treat in this section the situations where the two service providers work at different service rates $\mu_{1} \neq \mu_{2}$ but post the same prices $p_{1}=p_{2}=p$ and have symmetric locations $x_{2}=-x_{1}$. For utility functions satisfying Eq. (1), the dynamic boundary point $Y_{t}$ here obeys, $\forall t \in \mathbb{R}^{+}$: 


$$
Y_{t}= \begin{cases}\frac{c_{w}}{2 c_{t}}\left(\frac{N_{2}(t)}{\mu_{2}}-\frac{N_{1}(t)}{\mu_{1}}\right) & \text { if } c_{t} L \geqslant c_{w}\left|\left(\frac{N_{2}(t)}{\mu_{2}}-\frac{N_{1}(t)}{\mu_{1}}\right)\right|, \\ +\Delta & \text { if } c_{t} L<c_{w}\left(\frac{N_{2}(t)}{\mu_{2}}-\frac{N_{1}(t)}{\mu_{1}}\right), \\ -\Delta & \text { if } c_{t} L<c_{w}\left(\frac{N_{1}(t)}{\mu_{1}}-\frac{N_{2}(t)}{\mu_{2}}\right) .\end{cases}
$$

In view of Eq. (29), the dynamics is here driven by the difference between the normalized numbers of customers $\frac{N_{i}(t)}{\mu_{i}}, i=1,2$, waiting in the different queues. While we have used the approximation given by Eq. (11) in the symmetric case, we approximate here the dynamics implied by Eq. (29) with

$$
Y_{t}=\Delta f\left(\tilde{\gamma}\left(\frac{N_{2}(t)}{\mu_{2}}-\frac{N_{1}(t)}{\mu_{1}}\right)\right)
$$

where $f$ is a function satisfying the same hypothesis as in the symmetric case and

$$
\tilde{\gamma}:=\frac{c_{w}}{L c_{t}} .
$$

Note that $\tilde{\gamma}=\mu \gamma$ (with $\gamma$ being given by Eq. (12)). Following the same methodology used to derive Eqs. (6)-(8), it ensues that:

$$
\frac{N_{2}(t)}{\mu_{2}}-\frac{N_{1}(t)}{\mu_{1}}=\frac{\Lambda}{2} \int_{0}^{t}\left[\left(\frac{1}{\mu_{2}}-\frac{1}{\mu_{1}}\right)-\frac{1}{\Delta}\left(\frac{1}{\mu_{2}}+\frac{1}{\mu_{1}}\right) Y_{s}\right] \mathrm{d} s+\int_{0}^{t} \widetilde{V}\left(s, Y_{s}\right) \mathrm{d} B_{s},
$$

where, for Poisson arrival and service processes:

$$
\widetilde{V}\left(t, Y_{t}\right)^{2}=\frac{V_{1}\left(t, Y_{t}\right)^{2}}{\mu_{1}^{2}}+\frac{V_{2}\left(t, Y_{t}\right)^{2}}{\mu_{2}^{2}}=\frac{\Lambda Y_{t}}{2 \Delta}\left(\frac{1}{\mu_{1}^{2}}-\frac{1}{\mu_{2}^{2}}\right)+\frac{\Lambda}{2}\left(\frac{1}{\mu_{1}^{2}}+\frac{1}{\mu_{2}^{2}}\right)+\left(\frac{1}{\mu_{1}}+\frac{1}{\mu_{2}}\right) .
$$

Starting from Eq. (30) and following the lines used to derive Eqs. (11)-(17), we obtain:

$$
\mathrm{d} Y_{t}=\left[\frac{\frac{\tilde{\gamma} \Lambda \Delta}{2}\left(\frac{1}{\mu_{2}}-\frac{1}{\mu_{1}}\right)-\frac{\tilde{\gamma} \Lambda Y_{t}}{2}\left(\frac{1}{\mu_{1}}+\frac{1}{\mu_{2}}\right)}{\left(f^{-1}\right)^{\prime}\left(\frac{Y_{t}}{\Delta}\right)}\right] \mathrm{d} t+\frac{\Delta \tilde{\gamma} \widetilde{V}}{\left(f^{-1}\right)^{\prime}\left(\frac{Y_{t}}{\Delta}\right)} \mathrm{d} B_{t} .
$$

Setting $\mu_{1}=\mu_{2}=\mu$ in Eq. (33), we directly recover the dynamics valid in the symmetric case, given by Eq. (17). The stationary probability density function ensuing from the dynamics stated in Eq. (33) is given by

$$
P_{s}\left(\mu_{1}, \mu_{2} ; y\right)=\mathscr{N} \frac{\left(f^{-1}\right)^{\prime}\left(\frac{y}{\Delta}\right)}{\widetilde{V}(t, y)} \cdot \exp \left\{\frac{\Lambda}{\tilde{\gamma} \Delta^{2}} \int^{y}\left[\frac{\Delta\left(\frac{1}{\mu_{1}}-\frac{1}{\mu_{2}}\right)-u\left(\frac{1}{\mu_{1}}+\frac{1}{\mu_{2}}\right)}{\widetilde{V}(t, u)^{2}}\right]\left(f^{-1}\right)^{\prime}\left(\frac{u}{\Delta}\right) \mathrm{d} u\right\},
$$

where $\mathscr{N}$ is a normalization constant. Note that the structure of the dynamics obviously implies that $P_{s}\left(\mu_{1}, \mu_{2} ; y\right)=P_{s}\left(\mu_{2}, \mu_{1} ;-y\right)$. Remark that we consistently recover Eq. (19) when we fix $\mu_{1}=\mu_{2}=\mu$ in Eq. (34).

\subsubsection{Explicit illustration - asymmetric case}

For the particular choice $f(x)=\tanh (x)$, we find:

$$
P_{s}\left(\mu_{1}, \mu_{2} ; y\right)=\mathscr{N}\left(1+\frac{y}{\Delta}\right)^{-1-\frac{\beta-\alpha}{\xi-\delta}}\left(1-\frac{y}{\Delta}\right)^{-1-\frac{\beta+\alpha}{\xi+\delta}}(\delta \Delta+\xi y)^{\frac{2(\beta \xi \xi-\alpha \delta)}{\xi^{2}-\delta^{2}}-\frac{1}{2}}
$$

where

$$
\begin{aligned}
& \alpha=-\frac{\tilde{\gamma} \Lambda \Delta}{2}\left(\frac{1}{\mu_{1}}+\frac{1}{\mu_{2}}\right), \quad \beta=\frac{\tilde{\gamma} \Lambda \Delta}{2}\left(\frac{1}{\mu_{1}}-\frac{1}{\mu_{2}}\right), \quad \xi=\frac{\tilde{\gamma}^{2} \Lambda \Delta}{2}\left(\frac{1}{\mu_{1}^{2}}-\frac{1}{\mu_{2}^{2}}\right) \quad \text { and } \\
& \delta=\frac{\tilde{\gamma}^{2} \Lambda \Delta}{2}\left(\frac{1}{\mu_{1}^{2}}+\frac{1}{\mu_{2}^{2}}\right)+\tilde{\gamma}^{2} \Delta\left(\frac{1}{\mu_{1}}+\frac{1}{\mu_{2}}\right) .
\end{aligned}
$$

A sketch of the stationary distributions arising for heterogeneous services is given in Fig. 6. We observe that $P_{s}$ is shifted (and biaised) to the opposite side of the most effective server. This server clearly attracts more 

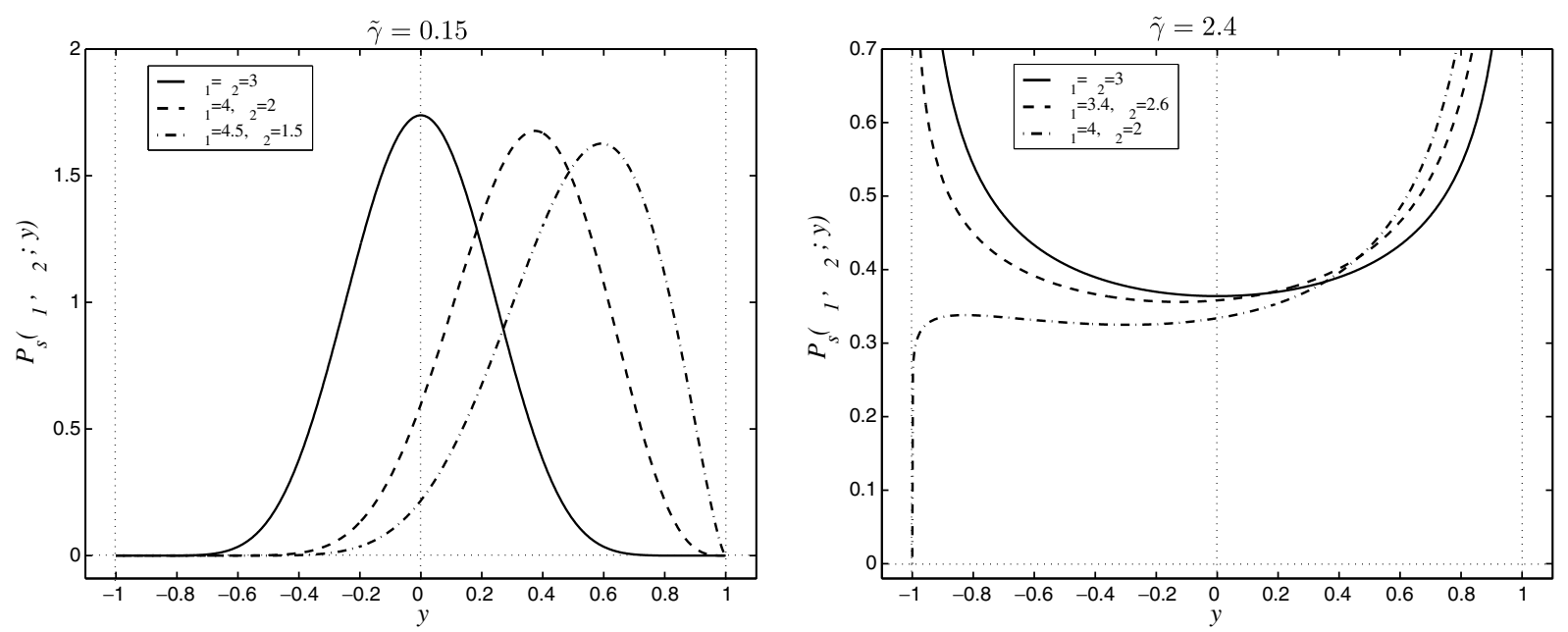

Fig. 6. Stationary probability density function of the time-dependent boundary position $Y_{t}$ for heterogeneous service rates $\mu_{1} \neq \mu_{2}$ and $f(x)=\tanh (x)$. Here, $\Delta=1$ and $\Lambda=5.7$. Left: $\tilde{\gamma}=0.15$ (Hotelling-like regime). Right: $\tilde{\gamma}=2.4$ (deadline type regime), note that the mixed boundary behavior of the dash-dot curve can only arise for asymmetric configurations.

customers than the slowest one. This illustrates the fact that the most effective server does enhance its market share.

Observe that for $\mu_{1}=\mu_{2}=\mu$ in Eq. (35), we consistently recover Eq. (23).

\subsection{Asymmetric positions and different prices}

When the two service providers are not symmetrically located with respect to the center of the market (i.e. $x_{1} \neq-x_{2}$ ), but have equal service rates and prices, the utility functions felt by the customers are modified such that the boundary position $Y_{t}$ obeys:

$$
Y_{t}= \begin{cases}\frac{c_{w}}{2 \mu c_{t}}\left(N_{2}(t)-N_{1}(t)\right)+\frac{x_{1}+x_{2}}{2} & \text { if } c_{t} L \geqslant|\widetilde{\Psi}|, \\ +\Delta & \text { if } c_{t} L<\widetilde{\Psi}, \\ -\Delta & \text { if } c_{t} L<-\widetilde{\Psi}\end{cases}
$$

Similarly, when the service providers differ only in their posted price (i.e. $p_{1} \neq p_{2}$ ), the time-dependent boundary position obeys:

$$
Y_{t}= \begin{cases}\frac{c_{w}}{2 \mu c_{t}}\left(N_{2}(t)-N_{1}(t)\right)+\frac{p_{2}-p_{1}}{2 c_{t}} & \text { if } c_{t} L \geqslant|\widehat{\Psi}| \\ +\Delta & \text { if } c_{t} L<\widehat{\Psi} \\ -\Delta & \text { if } c_{t} L<-\widehat{\Psi}\end{cases}
$$

where

$$
\widehat{\Psi}=\Psi\left(N_{1}(t), N_{2}(t), \mu, \mu, p_{1}, p_{2}\right)=p_{2}-p_{1}+\frac{c_{w}}{\mu}\left(N_{2}(t)-N_{1}(t)\right) .
$$

For both cases (or a combination of them), the addition of a static asymmetry contribution is required and the dynamics can be now approximated as

$$
Y_{t}=\Delta f\left(\gamma\left(N_{2}(t)-N_{1}(t)+\eta\right)\right) .
$$

Using the same methodology as for the symmetric case, we get:

$$
f^{-1}\left(\frac{Y_{t}}{\Delta}\right)=-\frac{\gamma \Lambda}{\Delta} \int_{0}^{t} Y_{s} \mathrm{~d} s+\gamma \int_{0}^{t} V\left(s, Y_{s}\right) \mathrm{d} B_{s}+\gamma \eta
$$


When taking the time-derivative of Eq. (39), the asymmetric contribution (i.e. $\gamma \eta$ ) disappears, and we get back to the symmetric dynamics given by Eq. (15). Hence for static asymmetry, the stationary probability density coincides with the symmetric case, given by Eq. (19). This should in fact not come as a surprise. Indeed, the static asymmetry manifests itself only during the transient regime. Starting with empty queues for both servers, the boundary point is initially located closer to the less attractive (in terms of price and/or position) server, implying thus a larger feeding rate to the most attractive one. The (static) lack of attractivity of one server will gradually be counterbalanced by a (dynamic) larger number of customers visiting the most attractive server. Asymptotically, the stationary regime for $Y_{t}$ behaves as in the symmetric case. Note however that, contrary to the symmetric case, the stationary queue contents will however not be equal anymore.

\section{Conclusion}

When alternative choices between services are offered to customers, several criteria enter into their final selection decision. There are namely static criteria such as posted prices and server locations as considered in the original Hotelling's model and dynamic aspects typified by the waiting times before service. It is intuitively clear that the negative aspects of the actual and/or perceived waiting times strongly affect the final customers' satisfaction and hence their decisions. As in generic situations the waiting time is an intrinsically random quantity, it is naturally described in the context of queueing theory. Focusing on the simple duopoly configuration, we study the (stochastic) dynamics of the frontier which defines the market partition. For heavy traffic regimes of the underlying queueing processes, the market frontier can be described by a (random) diffusion dynamics (i.e. a differential equation driven by a White Gaussian Noise) with a multiplicative noise (i.e. a state-dependent diffusion term). It is remarkable that the stationary probability measure characterizing the frontier process exhibits a noise-induced phase transition triggered by the values of the external control parameters (brand departure cost, waiting time cost, service rate and spatial separation between the servers). Note that multiplicative noise processes are not confined to physics, chemistry and biology domains where they first have been applied, they also naturally occur in operational research, in economics and more generally in social sciences. One of the most popular illustration is clearly found in financial mathematics - the Black-Scholes model, which is based on the geometric Brownian motion (hence a multiplicative noise process). Note however that contrary to the simple market sharing dynamics considered here, no noise-induced transition occurs in the financial context.

\section{Acknowledgements}

This work is partially supported by the "FNSR" (Fonds National Suisse pour la Recherche) and by the Fundaçao para a Ciênca e a Tecnologica FCT, FEDER/POCTI-SFA-1-219, Portugal. The authors would like to thank François Genoud for helpful discussions and two anonymous referees for their useful comments and constructive criticism.

\section{References}

[1] C. D'aspremont, J.J. Gabszewicz, J.F. Thisse, On hotelling's "stability in competition", Econometrica 47 (5) (1979) $1145-1150$.

[2] G.P. Cachon, P.T. Harker, Service Competition, Outsourcing and Co-Production in a Queueing Game, Preliminary Report. $<$ http:// knowledge.wharton.upenn.edu/papers/889.pdf> (March 1999).

[3] G.P. Cachon, P.T. Harker, Competition and outsourcing with scale economies, Management Science 48 (10) (2002) $1314-1333$.

[4] E. Çinlar, Introduction to Stochastic Processes, Prentice-Hall, Englewood Cliffs, 1975.

[5] R. Hassin, M. Haviv, To Queue or not to Queue, Kluwer Academic Publishers, 2003.

[6] W. Horsthemke, R. Lefever, Noise-Induced Transitions, second ed., Springer-Verlag, 2006.

[7] H. Hotelling, Stability in competition, The Economic Journal 39 (153) (1929) 41-57.

[8] J. Medhi, Stochastic Models in Queueing Theory, Academic Press, 1991, pp. 386-388.

[9] M. Montefiori, Spatial competition for quality in the market for hospital care, European Journal of Health Economics 6 (2005) 131135. 
[10] T. Puu, Hotelling's "ice cream dealers" with elastic demand, The Annals of Regional Science 36 (1) (2002) 1-17.

[11] H. Sanner, Instability in Competition: Hotelling Re-reconsidered, Diskussionsbeitrag Nr. 79, Universität Postdam, September 2005.

[12] E. Wong, The construction of a class of stationary markoff processes, in: Richard Bellman (Ed.), Sixteenth Symposium in Applied Mathematics - Stochastic Processes in Mathematical Physics and Engineering, American Mathematical Society, Providence, RI, 1964, pp. 264-276. 